\title{
Magnetic drug carriers: bright insights from light-responsive magnetic liposomes
}

\section{"...vector features converge to the same goal: conceiving an all-in-one platform for ultimate synergic cancer treatment and follow-up."}

Keywords: imaging • liposomes $\bullet$ magnetic carriers $\bullet$ magnetic hyperthermia - photodynamic therapy

We have witnessed rapid growth in magnetic drug carrier research in recent years. The relevance of drug carriers containing magnetic nanoparticles in the biomedical field has been highlighted due to the multiple properties of magnetic nanoparticles comprising magnetic actuation for magnetic drug targeting, contrast enhancement for MRI and heating dissipation for a therapeutic hyperthermia [1]. Individually or in combination, these properties render magnetic drug carriers unique tools bringing along current advances and future promise for drug delivery and beyond.

Drug targeting strategies aim to reverse nonselective drug biodistribution that otherwise is governed by drug physicochemical properties. They attempt to address the therapeutic agent to the site of interest by virtue of a drug carrier able to recognize and/or accumulate at the target. Magnetically assisted delivery of therapeutic agents at the site of interest, referred as magnetic drug targeting, is a promising strategy that has received growing interest [2-5]. In response to a magnetic field gradient generated by an externally positioned magnet, magnetic carriers are addressed to the site of interest where they are expected to release their therapeutic payload. By enhancing local drug concentration, an improved therapeutic outcome may be achieved while off-target side effects in healthy tissues may be prevented [6].

In addition to magnetic actuation, imaging and heating features are remarkable assets of magnetic nanoparticles giving rise to theranostic strategies for combined therapy in an ultimate all-in-one nanoplatform. Such multiple functionalities resonate with the need to enable real-time imaging of magnetic carriers for precise tumor addressing and to validate nanosystem accumulation at the tumor. Moreover, the integration of heating capability and/or a chemotherapy payload are promising strategies to tackle tumors by distinct cytotoxic approaches so that tumor cells resistant to one of them may be stricken by the other. In addition to complementarity, synergism may also play a chief role as the cytotoxic mechanism from each strategy may enhance one another, as it will be further commented below.

In order to enable a magnetic drug carrier to exert all its tasks, the design of magnetic drug carriers deserves particular attention. Therapeutic agents may be coupled to the functionalized surface of magnetic nanoparticles by virtue of electrostatic or covalent bonds. Although this represents a straightforward approach, drug release may be hampered in the case of strong covalent bonds or a burst release previous to target reaching may result from weak electrostatic drug binding. Alternatively, drugs may be encapsulated using an inorganic or organic coating allowing drug loading and release, which additionally enable nanoparticle stabilization by modulating surface properties [7]. Concerning inorganic shells, the most frequent ones regard silica and zeolites, which present high porosity and specific surface area for interacting with therapeutic agents [8]. In addition to inorganic coating, polymer or lipid-based


Amanda KA Silva

Laboratoire Matière et Systèmes Complexes, UMR 7057, CNRS

\& University Paris Diderot, 75205 Paris cedex 13, France



Christine Ménager Laboratoire PHENIX, Sorbonne Universités, UPMC, University Paris 06, UMR CNRS 8234, 4 place Jussieu 75005 Paris, France

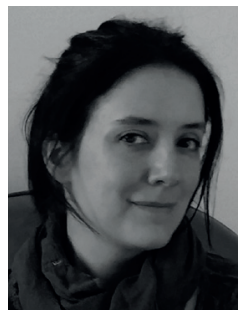

Claire Wilhelm

Author for correspondence: Laboratoire Matière et Systèmes Complexes, UMR 7057, CNRS \& University Paris Diderot, 75205 Paris cedex 13, France

wilhelm@univ-paris-diderot.fr
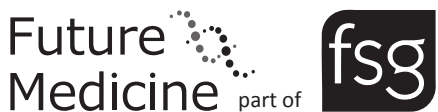
organic shells have been widely investigated. Polymeric carriers endowed with magnetic nanoparticles have been designed using chitosan, polyethylene glycol and dextran, just to name a few. Core-shell polymer-coated magnetic nanoparticles were successfully designed and also micelles constituted by amphiphilic block copolymers were conceived to encapsulate therapeutic agents and magnetic nanoparticles. In parallel, lipid-based magnetic carriers, namely magnetoliposomes, have also been investigated.

"...we demonstrated that the association
of photodynamic therapy with magnetic
hyperthermia by virtue of the photosensitizer-
loaded ultramagnetic liposomes resulted in
complete tumor regression in a murine model."

Liposomes represent a versatile drug carrier matching high biocompatibility and the ability to enclose polar drugs in its inner aqueous cavity and hydrophilic or apolar therapeutic payloads in the lipid bilayer [9]. Liposomes have been investigated for encapsulating magnetic nanoparticles and several drug molecules [10], as for instance, doxorubicin, antiestrogen drugs and muscle relaxants, just to name a few. Concerning the encapsulation of magnetic nanoparticles, the main issue is to achieve a high degree of loading. The magnetization per liposome should be sufficient to counterbalance the blood flow in vivo for magnetic targeting or to obtain a therapeutically competent temperature elevation under alternative magnetic field for magnetic hyperthermia. We have demonstrated that the reverse phase evaporation technique enables much higher internal aqueous loading (almost a 100-fold) in comparison with more classical thin film hydration strategies. It first consists in the formation of a water-in-oil emulsion where the water phase carries the nanoparticles and the organic phase encloses the lipids for the future bilayer. Slow evaporation of the organic solvent under reduced pressure then converts the system into a viscous gel, finally resulting in an aqueous suspension containing the liposomes. The Fe/lipids ratio can then reach values in the order of $100 \mathrm{~mol} \mathrm{Fe} /$ mol lipids, corresponding to an estimated number of 10,000 nanoparticles per liposome.

These ultramagnetic liposomes are thus appealing candidates to deliver an efficient therapeutic magnetic hyperthermia at a tumoral site. In a recent study, we successfully enclosed the 5,10,15,20-tetra(m-hydroxyphenyl)chlorin photosensitizer, already used in the clinics under the trade name of FOSCAN for photodynamic therapy, within the bilayer of ultramagnetic liposomes [11]. Double functionality stemmed from the double cargo: the magneto-photo-responsive lipo- somes generated highly cytotoxic reactive oxygen species under laser excitation and produced heat under alternative magnetic field stimulation, coupling photodynamic therapy to magnetic hyperthermia for a synergic effect.

The assets of such a combined therapy and the resulting synergy rely on the intrinsic features, the strengths and the weaknesses of each modality. Photodynamic therapy is based on the combined action of three components: a photosensitizer, light and oxygen. Upon irradiation at an appropriate wavelength $(650 \mathrm{~nm}$ for FOSCAN), the photosensitizer is activated and transfers its excited-state energy to surrounding oxygen. This results in the production of reactive oxygen species, such as singlet oxygen, which will induce cell death [12]. In addition to singlet oxygen production, photosensitizers emit fluorescence, which enables both detection and imaging of the drug. Therefore, photodynamic therapy is currently considered an appealing theranostic modality for malignant diseases [13], which has already made its way to the clinics. However, photodynamic therapy still suffers from suboptimal concentration at tumor site, off-target side effects due to unspecific intake in healthy tissues and mild singlet oxygen production due to local hypoxia [14-17]. Coupling photodynamic therapy with other therapeutic modalities, such as magnetic hyperthermia may represent a synergistic approach in the attempt to circumvent current limitations of both strategies. On one hand, hyperthermia will increase local blood flow and oxygenation, which may reduce hypoxia-related limitation [18]. On the other hand, photodynamic therapy may enhance the hyperthermia effect by weakening mechanisms involved in heat shock cell defense [19].

Indeed, we demonstrated that the association of photodynamic therapy with magnetic hyperthermia by virtue of the photosensitizer-loaded ultramagnetic liposomes resulted in complete tumor regression in a murine model [11]. Additionally, the magnetic content of the liposomes also enabled the in vivo distribution follow-up of the carrier by MRI. Collectively, the reported results converged to attest that hybrid liposomes can successfully integrate advanced functionality, for example, light responsiveness, magnetic responsiveness and image tracking, going beyond simple drug delivery. In a parent study, we demonstrated the coloading of a photosensitizer and magnetic nanoparticles in a biogenic vesicle [20], produced by cells in response to a biological, chemical or mechanical stress. To translate extracellular vesicles into vesicular magnetic drug delivery vectors, the 'synthesis' approach consists of loading cells with different agents (here the FOSCAN photosensitizer and magnetic nanoparticles), and triggering (e.g., by starvation) the release of 
vesicles, which will carry inside the internalized drug and nanoparticle agents. The resulting vesicle is thus the biogenic reflection of its synthetic counterpart, the magneto-photo-responsive liposome. In both cases, vector features converge to the same goal: conceiving an all-in-one platform for ultimate synergic cancer treatment and follow-up. A next frontier is to investigate therapy in orthotopic tumor in more relevant animal models. Additionally, the prospect of intravenous administration coupled to magnetically assisted drug targeting seems very exciting. Indeed, the outstanding magnetic nanoparticle load of the designed ultramagnetic liposomes is expected to favor magnetic capture

\section{References}

1 Pankhurst Q, Thanh N, Jones S, Dobson J. Progress in applications of magnetic nanoparticles in biomedicine. J. Phys. D Appl. Phys. 42, 224001 (2009).

2 Mikhaylov G, Mikac U, Magaeva AA et al. Ferri-liposomes as an MRI-visible drug-delivery system for targeting tumours and their microenvironment. Nat. Nanotechnol. 6, 594-602 (2011).

3 Arruebo M, Fernández-Pacheco R, Ibarra MR, Santamaría J. Magnetic nanoparticles for drug delivery. Nano Today 2, 22-32 (2007).

$4 \quad$ Namiki Y, Namiki T, Yoshida H et al. A novel magnetic crystal-lipid nanostructure for magnetically guided in vivo gene delivery. Nat. Nanotechnol. 4, 598-606 (2009).

5 Gultepe E, Reynoso FJ, Jhaveri A et al. Monitoring of magnetic targeting to tumor vasculature through MRI and biodistribution. Nanomedicine (Lond.) 5, 1173-1182 (2010).

6 Pankhurst QA, Connolly J, Jones S, Dobson J. Applications of magnetic nanoparticles in biomedicine. J. Phys. D Appl. Phys. 36, R167 (2003).

7 Sun C, Lee JSH, Zhang M. Magnetic nanoparticles in MR imaging and drug delivery. Adv. Drug Deliv. Rev. 60, 1252-1265 (2008).

8 Durán JDG, Arias JL, Gallardo V, Delgado AV. Magnetic colloids as drug vehicles. J. Pharm. Sci. 97, 2948-2983 (2008).

9 Soenen SJ, Velde GV, Ketkar-Atre A, Himmelreich U, De Cuyper M. Magnetoliposomes as magnetic resonance imaging contrast agents. Wiley Interdiscip. Rev. Nanomed. Nanobiotechnol. 3, 197-211 (2011).

10 Pattni BS, Chupin VV, Torchilin VP. New developments in liposomal drug delivery. Chem. Rev. doi:10.1021/acs. chemrev.5b00046 (2015) (Epub ahead of print).

11 Di Corato R, Béalle G, Kolosnjaj-Tabi J et al. Combining magnetic hyperthermia and photodynamic therapy for tumor ablation with photoresponsive magnetic liposomes. ACS Nano 9, 2904-2916 (2015). and accumulation at the target. The perspective of these achievements would be of great benefit in order to advance in the long path toward clinical translation.

\section{Financial \& competing interests disclosure}

The authors have no relevant affiliations or financial involvement with any organization or entity with a financial interest in or financial conflict with the subject matter or materials discussed in the manuscript. This includes employment, consultancies, honoraria, stock ownership or options, expert testimony, grants or patents received or pending, or royalties.

No writing assistance was utilized in the production of this manuscript.

12 Robertson CA, Evans DH, Abrahamse H. Photodynamic therapy (PDT): a short review on cellular mechanisms and cancer research applications for PDT. J. Photochem. Photobiol. B 96, 1-8 (2009).

13 Lovell JF, Liu TWB, Chen J, Zheng G. Activatable photosensitizers for imaging and therapy. Chem. Rev. 110, 2839-2857 (2010).

14 Das K, Dube A, Gupta P. A spectroscopic study of photobleaching of Chlorin p 6 in different environments. Dyes Pigm. 64, 201-205 (2005).

15 Huang P, Li Z, Lin J et al. Photosensitizer-conjugated magnetic nanoparticles for in vivo simultaneous magnetofluorescent imaging and targeting therapy. Biomaterials 32, 3447-3458 (2011).

16 Senge MO, Brandt JC. Temoporfin (Foscan ${ }^{\circledR}, 5,10,15$, 20-tetra (m-hydroxyphenyl) chlorin) - a second-generation photosensitizer. Photochem. Photobiol. 87, 1240-1296 (2011).

17 Busch TM, Hahn SM, Evans SM, Koch CJ. Depletion of tumor oxygenation during photodynamic therapy: detection by the hypoxia marker EF3 [2-(2-nitroimidazol-1[H]yl)-N-(3,3,3-trifluoropropyl)acetamide]. Cancer Res. 60, 2636-2642 (2000).

18 Yanase S, Nomura J, Matsumura Y, Nagata T, Fujii T, Tagawa T. Synergistic interaction of 5-aminolevulinic acid-based photodynamic therapy with simultaneous hyperthermia in an osteosarcoma tumor model. Int. J. Oncol. 29, 365-373 (2006).

19 Frank J, Lambert C, Biesalski HK, Thews O, Vaupel P, Kelleher DK. Intensified oxidative and nitrosative stress following combined ALA-based photodynamic therapy and local hyperthermia in rat tumors. Int. J. Cancer 107, 941-948 (2003).

20 Silva AK, Kolosnjaj-Tabi J, Bonneau S et al. Magnetic and photoresponsive theranosomes: translating cell-released vesicles into smart nanovectors for cancer therapy. ACS Nano 7, 4954-4966 (2013). 